\title{
Self-Limited COVID-19 in a Patient with Artemis Hypomorphic SCID
}

\author{
Stanislaw J. Gabryszewski ${ }^{1}$. Ross N. England ${ }^{2}$. Di Sun ${ }^{1} \cdot$ Tatiana Londoño Gentile $^{3}$. William Hochgertel ${ }^{3}$. \\ Soma Jyonouchi ${ }^{1} \cdot$ Michael Silverman $^{2,4} \cdot$ Theoklis Zaoutis $^{2} \cdot$ Kathleen E. Sullivan ${ }^{1,4} \cdot$ Sarah E. Henrickson $^{1,4,5}$
}

Received: 16 March 2021 / Accepted: 1 July 2021 / Published online: 21 August 2021

(C) The Author(s), under exclusive licence to Springer Science+Business Media, LLC, part of Springer Nature 2021

To the Editor,

The viral etiology of coronavirus disease 2019 (COVID-19), caused by the severe acute respiratory syndrome coronavirus 2 (SARS-CoV-2), led to initial conjecture that $\mathrm{T}$ cell and B cell responses would be essential for disease resolution $[1,2]$. However, this has become less clear over time. The clinical manifestations of COVID-19 range from asymptomatic disease to multi-organ failure and death. COVID-19 is also associated with presumed post-infectious phenomena, including the multisystem inflammatory syndrome in children (MIS-C). While established risk factors for severe COVID-19 include advanced age, obesity, heart disease, and cancer, the extent to which specific inborn errors of immunity (IEI) predispose to severe COVID-19 remains an area of active inquiry [2,3].

SARS-CoV-2 evokes both innate and adaptive immune responses. Highlighting the importance of innate immunity, factors associated with severe disease include genetic defects and autoantibodies that disrupt interferon (IFN) signaling $[4,5]$. Humoral immunity in COVID-19 is characterized in

Sarah E. Henrickson

henricksons@chop.edu

1 Division of Allergy and Immunology, Department of Pediatrics, Children's Hospital of Philadelphia, University of Pennsylvania Perelman School of Medicine, Philadelphia, PA, USA

2 Division of Infectious Diseases, Department of Pediatrics, Children's Hospital of Philadelphia, University of Pennsylvania Perelman School of Medicine, Philadelphia, PA, USA

3 Department of Pediatrics, Children's Hospital of Philadelphia, University of Pennsylvania, Perelman School of Medicine, Philadelphia, PA, USA

4 Institute for Immunology, University of Pennsylvania Perelman School of Medicine, Philadelphia, PA 19104, USA

5 Department of Microbiology, Perelman School of Medicine, University of Pennsylvania, Philadelphia, PA, USA part by production of neutralizing antibodies, most notably targeting the SARS-CoV-2 Spike protein that allows for viral entry into host cells [6]. Coordination of humoral immunity with $\mathrm{CD}^{+}$and $\mathrm{CD} 8^{+} \mathrm{T}$ cell responses has been associated with disease resolution [7]. Severe infection may cause lymphopenia, CD8 T cell immune dysregulation, and altered B cell differentiation $[8,9]$. Importantly, interpretations about the relative contributions of specific immune responses to disease resolution versus pathology must be made with caution given the lack of baseline, pre-illness samples in published affected patients. These relationships can be better parsed by studying the impact of COVID-19 on patients with IEI with known baseline immune states.

Severe combined immunodeficiency (SCID) is a group of rare disorders caused by mutations in genes important for lymphocyte development. One of the causative genes, DCLRE1C, encodes the $\mathrm{V}(\mathrm{D}) \mathrm{J}$ recombination endonuclease Artemis. Artemis deficiency impairs the generation of antigenic receptor diversity, which affects $\mathrm{T}$ and $\mathrm{B}$ cells, but not NK cells. Untreated SCID causes increased susceptibility to opportunistic infections, faltering growth, and lethality in infancy. In hypomorphic (or "leaky") SCID, T cell numbers and function are reduced but not absent, and the resulting clinical phenotype is more subtle than in classic SCID. Limited data exist on COVID-19 outcomes in patients with classic and hypomorphic SCID [2, 3]. While disproportionately high rates of lethality have been documented in SCID patients in an analysis of IEI patients with COVID-19, successful clinical outcomes have been observed in select cases $[3,10,11]$.

A 14-month-old female with Artemis hypomorphic SCID presented to our institution with fever and cough following recent exposure to family members with COVID-19. Her past medical history was notable for uncomplicated term birth and presumptive positive SCID newborn screen ( $\mathrm{T}$ cell receptor excision circle count $<25$ ). Flow cytometry of key cell subsets was as follows (cells per microliter): $\mathrm{CD} 3^{+} 908$ (reference 2300-7000), CD4 $4^{+} 775$ (reference 1700-5300), $\mathrm{CD}^{+} 96$ (reference $400-1700$ ), $\mathrm{CD} 19^{+} 12$ (reference 


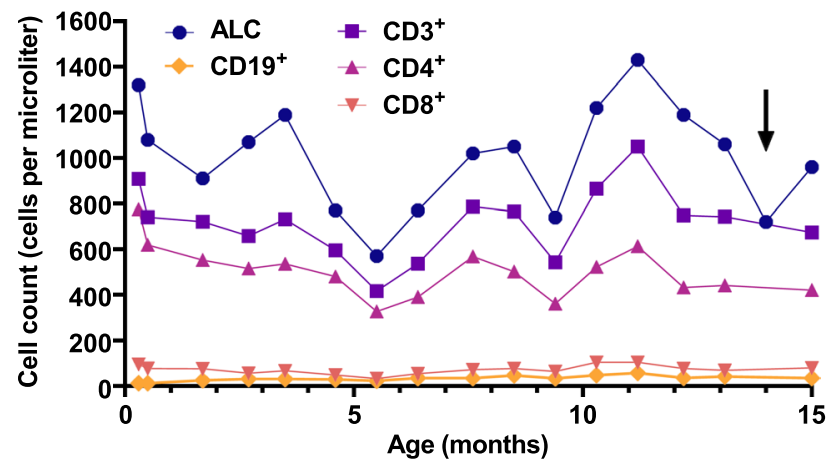

Fig. $1 \mathrm{~T}$ cell and B cell counts since birth. Shown are absolute counts (cells per microliter) for lymphocytes, $\mathrm{CD}^{+} \mathrm{T}$ cells, $\mathrm{CD} 4^{+} \mathrm{T}$ cells, $\mathrm{CD}^{+} \mathrm{T}$ cells, and $\mathrm{CD} 19^{+} \mathrm{B}$ cells over time (age in months). Time of COVID-19 diagnosis is indicated by an arrow. ALC, absolute lymphocyte count

600-1900), NK 342 (reference 200-1400), CD4 ${ }^{+} / \mathrm{CD} 45 \mathrm{RA}$ naïve 734 (reference $41-1121$ ), and $\mathrm{CD} 4 \%$ CD $45 \mathrm{RO}$ memory 57 (reference 153-582), collectively demonstrating $\mathrm{T}$ lymphopenia with elevated CD4-to-CD8 ratio and appropriate naïve-to-memory $\mathrm{T}$ cell ratio for age (12.8:1), near-absent $\mathrm{B}$ cells, and normal NK cell numbers. Absolute counts (cells per microliter) of lymphocyte subsets one month prior to presentation were $\mathrm{CD}^{+} 743$ (reference $1600-6700$ ), $\mathrm{CD} 4^{+}$ 441 (reference 1000-4600), $\mathrm{CD}^{+} 69$ (reference 400-2100), $\mathrm{CD}_{1} 9^{+} 42$ (reference 600-2700), and NK 236 (reference 200-1200) (Fig. 1). Additionally, lymphocyte stimulation to phytohemagglutinin (PHA) was decreased ( $46 \%$ of control), and TCR V $\beta$ spectratyping revealed a polyclonal $\mathrm{T}$ cell receptor (TCR) repertoire. PCR-based maternal engraftment testing at 3 months of age was normal. Immunoglobulin levels (milligrams per deciliter) at 2 weeks of life were $\operatorname{Ig} \mathrm{A}<6, \operatorname{IgE}<5, \operatorname{IgG} 697$, and $\operatorname{IgM} 6$. Genetic testing (Primary Immunodeficiency Panel, Invitae) revealed compound heterozygous mutations in Artemis-encoding DCLRE1C, with a maternally inherited pathogenic deletion in exons 1-3 and a paternally inherited suspected pathologic variant (NM 001033855.2; c.632G > C, p.Gly211Ala; CADD score 27.9; minor allele frequency $10^{-7}$ ). To date, a fully matched hematopoietic cell transplant (HCT) donor has not been identified. Therefore, the patient is managed with infection prophylaxis (trimethoprim-sulfamethoxazole, fluconazole, and intravenous immunoglobulin) and undergoes routine immunologic lab surveillance.

The patient presented on day 2 of illness with fever $\left(\mathrm{T}_{\max } 38.7{ }^{\circ} \mathrm{C}\right)$, cough, rhinorrhea, and loose stools following exposure to two family members with polymerase chain reaction (PCR)-confirmed SARS-CoV-2 infection. The patient's SARS-CoV-2 nasopharyngeal PCR test was positive, and she was admitted to our institution's COVID19 isolation unit. Her workup was notable for lymphopenia
Table 1 Proinflammatory cytokine panel results on day 3 of illness

\begin{tabular}{lll}
\hline Cytokine & $\begin{array}{l}\text { Concentration } \\
(\mathrm{pg} / \mathrm{ml})\end{array}$ & $\begin{array}{l}\text { Reference } \\
\text { range (pg/ } \\
\mathrm{ml})\end{array}$ \\
\hline IFN- $\gamma$ & 40.5 & $0-6.5$ \\
IL-1 $\beta$ & $<2.5$ & $0-2.5$ \\
IL-2 & $<6.5$ & $0-6.5$ \\
IL-4 & $<1.5$ & $0-1.5$ \\
IL-6 & $<3.5$ & $0-3.5$ \\
IL-8 & 19.7 & $0-10$ \\
IL-10 & $<2.0$ & $0-2$ \\
IL-12p70 & $<2.5$ & $0-2.5$ \\
IL-13 & $<2.5$ & $0-2.5$ \\
TNF- $\alpha$ & 5.8 & $0-3.5$ \\
\hline
\end{tabular}

(absolute lymphocyte count decreased to 720 cells per microliter from 1060 one month prior) with concomitant elevated levels of the cytokines IFN- $\gamma$, IL- 8 , and TNF- $\alpha$ (Table 1). C-reactive protein level was normal, and blood culture was negative. Collectively, the patient's presentation and diagnostic findings were consistent with acute COVID19. Infectious disease was consulted and did not recommend antiviral treatment or convalescent plasma given the patient's mild symptoms. The patient was closely observed for $36 \mathrm{~h}$ and discharged after showing clinical improvement with adequate enteral intake and balanced fluid status. Repeat SARS-CoV-2 PCR studies were not performed to exclude possibility of persistent virus shedding given the patient's rapid clinical improvement.

The mild severity of the patient's infection may be in part attributable to her intact innate immune function, a well-documented modulator of COVID-19 severity [4, 5]. However, it is remarkable that the patient experienced full clinical resolution of COVID-19 despite having persistent T lymphopenia and near-absence of B cells, supporting ongoing questions into the relative importance of $\mathrm{T}$ and $\mathrm{B}$ cell responses in overcoming SARS-CoV-2 infection. In a recent analysis of immune responses in acutely ill and convalescent subjects, resolution of COVID-19 was associated with coordinated $\mathrm{T}$ and $\mathrm{B}$ cell responses [7]. It is conceivable that the patient's polyclonal TCR repertoire was sufficiently diverse to induce effective $T$ cell immunity, even with fewer total T lymphocytes. Further, as advanced age is linked to severity of COVID-19 [6], it is possible the patient's young age contributed to her favorable clinical outcome, though the underlying mechanisms causing improved outcomes in the young remain active areas of investigation.

The patient's excellent clinical outcome despite having essentially absent B cells aligns with the observation that the presence of SARS-CoV-2-specific neutralizing antibodies does not correlate with reduced disease severity [7]. 
Following reports of mild, self-limited COVID-19 in two patients with agammaglobulinemia, it has been postulated that B cells may not be necessary for resolution of infection [12]. Consistent with this, a mild clinical course was also observed in COVID-19 patients treated with the biologics ocrelizumab and rituximab, which selectively deplete B cells [13]. Interestingly, a series of patients with common variable immunodeficiency (CVID), in which B cells are dysfunctional but not absent, experienced a more severe clinical course, suggesting a role for dysregulated B cell responses in COVID-19 immunopathology [12].

In summary, despite significant underlying T lymphopenia and absence of B cells, this patient with hypomorphic SCID experienced mild COVID-19 disease that resolved without pharmacologic therapy. The dynamics of immune responses in COVID-19 and their contributions to the balance between disease pathology and disease resolution remain incompletely understood. To this end, efforts focused on targeted treatment strategies will undoubtedly benefit from investigations of COVID-19 outcomes in patients with defined immunologic deficits.

Acknowledgements We thank the patient's primary caregivers and the family for their permission to share this case report with the scientific community. We acknowledge all healthcare workers who were involved in the care of this patient during her hospitalization.

Author Contribution SJG and SEH conceived the original version of this manuscript. SJG, RNE, DS, TLG, WH, SJ, MS, TZ, KES, and SEH contributed to subsequent versions of the manuscript and arrangement of figures. All authors approved of the final version of the manuscript. SJG, RNE, TLG, WH, MS, TZ, KES, and SEH were directly involved in the clinical management of this patient, as described in this manuscript. DS and SJ provided pertinent diagnostic information about the patient and are directly involved in ongoing clinical care of the patient.

Funding Financial support for this project was provided by NIAID K08AI135091 (SEH). All other authors had no funding that they wanted to share.

Data Availability The material described in this manuscript has not been published elsewhere.

Code Availability Not applicable.

\section{Declarations}

Conflict of Interest The authors declare no competing interests.

\section{References}

1. Poland GA, Ovsyannikova IG, and Kennedy RB. SARS$\mathrm{CoV}-2$ immunity: review and applications to phase 3 vaccine candidates. Lancet. 2020;396(10262):1595-1606. https://doi.org/ 10.1016/S0140-6736(20)32137-1.

2. Meyts I, Bucciol G, Quinti I, Neven B, Fischer A, Seoane E, et al. Coronavirus disease 2019 in patients with inborn errors of immunity: an international study. J Allergy Clin Immunol. 2020;147(2):520-531. https://doi.org/10.1016/j.jaci.2020.09.010.

3. Delavari S, Abolhassani H, Abolnezhadian F, Babaha F, Iranparast $\mathrm{S}$, Ahanchian $\mathrm{H}$, et al. Impact of SARS-CoV-2 pandemic on patients with primary immunodeficiency. J Clin Immunol. 2021;41(2):345-355.https://doi.org/10.1007/ s10875-020-00928-x.

4. Zhang Q, Bastard P, Liu Z, Le Pen J, Moncada-Velez M, Chen $\mathrm{J}$, et al. Inborn errors of type I IFN immunity in patients with life-threatening COVID-19. Science. 2020;370(6515):eabd4570. https://doi.org/10.1126/science.abd4570.

5. Bastard P, Rosen LB, Zhang Q, Michailidis E, Hoffmann HH, Zhang Y, et al. Autoantibodies against type I IFNs in patients with life-threatening COVID-19. Science. 2020;370(6515):eabd4585. https://doi.org/10.1126/science.abd4585.

6. Sette A and Crotty S. Adaptive immunity to SARS-CoV-2 and COVID-19. Cell. 2021;184(4):861-880. https://doi.org/10.1016/j. cell.2021.01.007.

7. Moderbacher CR, Ramirez SI, Dan JM, Grifoni A, Hastie KM, Weiskopf D, et al. Antigen-specific adaptive immunity to SARSCoV-2 in acute COVID-19 and associations with age and disease severity. Cell. 2020;183(4):996-1012.e19. https://doi.org/10. 1016/j.cell.2020.09.038.

8. Vabret N, Britton GJ, Gruber C, Hegde S, Kim J, Kuksin M, et al. Immunology of COVID-19: current state of the science. Immunity. 2020;52(6):910-941. https://doi.org/10.1016/j.immuni.2020. 05.002.

9. Mathew D, Giles JR, Baxter AE, Oldridge DA, Greenplate AR, $\mathrm{Wu}$ JE, et al. Deep immune profiling of COVID-19 patients reveals distinct immunotypes with therapeutic implications. Science. 2020;369(6508):eabc8511. https://doi.org/10.1126/science. abc8511.

10. Al-Qahtani KM, Alhamdan AS, Alblowi WF, Al-Amri MM, Almalki MH. Successful handling of COVID-19 in young patient with SCID due to CARMIL2 (RLTPR) deficiency: a case report and review of literature. J Genom Gene Study. 2020;S(1):03.

11. van Oers NS, Hanners NW, Sue PK, Aquino V, Li QZ, Schoggins JW, et al. SARS-CoV-2 infection associated with hepatitis in an infant with X-linked severe combined immunodeficiency. Clin Immunol. 2021;224:108662. https://doi.org/10.1016/j.clim.2020. 108662.

12. Quinti I, Lougaris V, Milito C, Cinetto F, Pecoraro A, Mezzaroma I, et al. A possible role for B cells in COVID-19? Lesson from patients with agammaglobulinemia. J Allergy Clin Immunol. 2020;146(1):211-213.e4. https://doi.org/10.1016/j.jaci.2020.04. 013.

13. Fung M and Babik JM. COVID-19 in immunocompromised hosts: what we know so far. Clin Infect Dis. 2021;72(2):340-350. https:// doi.org/10.1093/cid/ciaa863.

Publisher's Note Springer Nature remains neutral with regard to jurisdictional claims in published maps and institutional affiliations. 